\title{
THE COGNITIVE AND NEUROANATOMICAL UNDERPINNINGS OF DESTINATION MEMORY
}

The ability to remember the destination to whom a piece of information has been addressed (e.g., did I tell you about the weekend?) has been labelled destination memory. Although this topic has been relatively scarcely studied, recent studies support the notion that destination recall can be the subject of important distortions in healthy younger and older adults and in individuals with Alzheimer's disease. This research also links destination recall to several cognitive domains such as episodic memory, executive function, and self-referential processes (e.g., did / tell you about the weekend?). The present review aims to assemble these findings into a comprehensive framework and shed light onto potential neuroanatomical underpinnings of destination memory, thus providing a promising venue for future exploration and research. - Destination memory $\cdot$ Episodic memory $\cdot$ Medial temporal cortex $\cdot$ Precuneus $\cdot$ Prefrontal cortex

\section{Destination memory: definition and cognitive underpinnings}

In our daily lives, we are constantly relaying information to people in our environment, such as friends, family, or strangers. Remembering to whom information has been previously outputted, referred to as destination memory, allows for successful associations between messages and their receiver(s), thus enhancing communicative efficacy and, consequently, our daily interactions with others [1-3]. Research shows that destination recall (i.e., remembering to whom information was provided) is difficult and can be subject to distortions in several populations. In this area of research, Gopie and MacLeod [3] asked young participants to tell (destination condition) and receive (source condition) facts to and from pictures of celebrity faces. In a subsequent recognition task, the participants had to decide to/from which face they had previously emitted/ received the facts. These procedures showed more errors on destination than on source recognition. Subsequent work showed that

*E-mail:mohamad.el-haj@univ-montp3.fr
Keywords destination memory is additionally hampered in normal aging [4,5] and impaired, more than source memory, in Alzheimer's disease $[1,2,6]$. The vulnerability of destination memory has been attributed to the assumption that

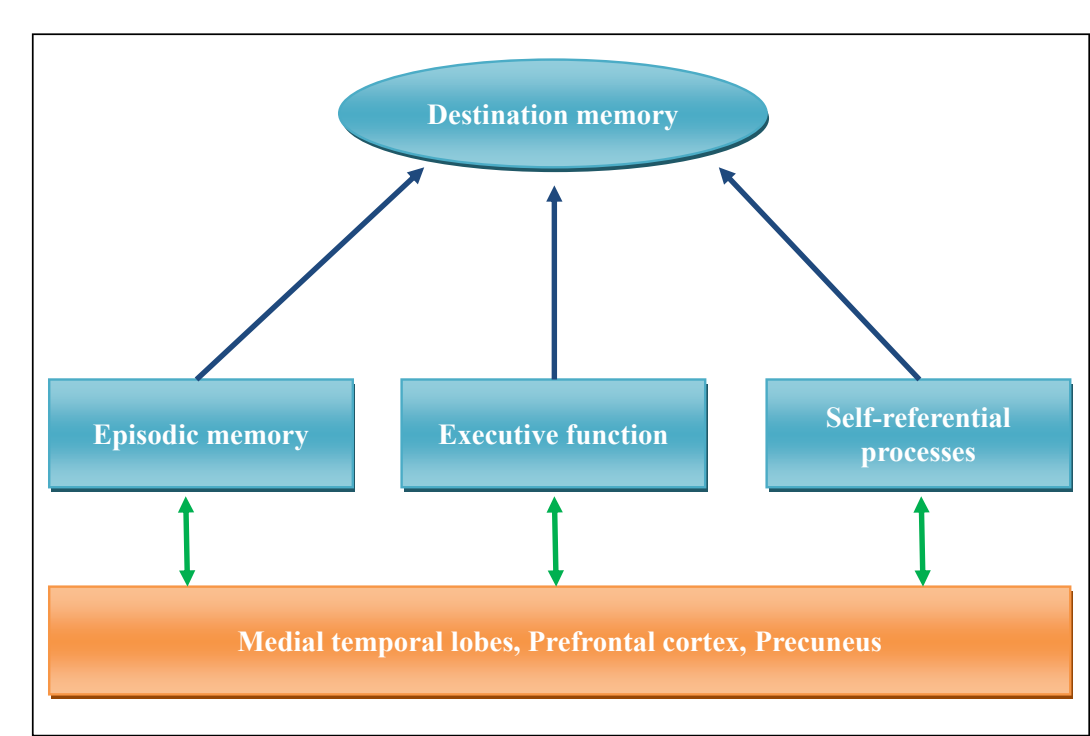

Figure 1. Destination memory seems to require different cognitive resources, such as episodic memory, executive
function, and self-referential processes; cognitive resources that are dependent on medial temporal

Figure 1. Destination memory seems to require different cognitive resources, such as episodic memory, executive
function, and self-referential processes; cognitive resources that are dependent on medial temporal lobes, prefrontal lobes and precuneus. on cognitive resources, such as episodic processing, executive function, and on selfreferential processes, such as self-monitoring $[1-3,5,6]$ (see Figure 1).
Mohamad El Haj ${ }^{1,2 *}$

Philippe Allain ${ }^{3,4}$

Roy P. C. Kessels ${ }^{5,6,7}$

Epsylon LAboratory, EA 4556,

University Montpellier III, Montpellier, France

${ }^{2}$ Research Unit on Cognitive and Affective Sciences,

Department of Psychology,

University of North of France

${ }^{3}$ LUNAM Université, Laboratoire de Psychologie

des Pays de la Loire (EA 4638),

Université de Nantes et Angers, France

${ }^{4}$ Centre Mémoire de Ressources et de Recherches, CHU Angers, France

${ }_{5}^{5}$ Donders Institute for Brain,

Cognition and Behaviour, Radboud University

Nijmegen, The Netherlands

${ }^{6}$ Vincent van Gogh Institute for Psychiatry, Korsakoff Clinic, Venray, The Netherlands

${ }^{7}$ Department of Medical Psychology, Radboud University Nijmegen Medical Centre, Nijmegen, The Netherlands

Received 04 June 2014 accepted 08 June 2014

this destination memory may draw heavily 


\subsection{Destination memory and episodic recall}

With respect to the cognitive processes underlying destination memory, an important role has been suggested for episodic memory recall in general. For instance, El Haj et al. [6] reported significant correlations between destination memory deterioration and perturbation of autonoetic reliving in individuals with Alzheimer's disease. The latter ability, or the subjective experience characterizing episodic recall, was evaluated with the "Remember/Know" paradigm, a paradigm allowing evaluation of the ability to recall specific episodic elements [7-9]. Further evidence about the link between destination memory deterioration and episodic decline was found by El Haj et al. [6] who observed a significant correlation between destination memory and the Grober and Buschke's task [10], a standard episodic memory testing protocol, a correlation that was observed in both healthy older adults and individuals with Alzheimer's disease.

Destination memory allows for making judgments about the context in which an event occurred (e.g., to whom a piece of information was sent), which specifies contextual features of a particular episode and contributes to discriminating a targeted episode from similar events. Destination processing thus requires integrative processes, that is, binding items to their context destinations, which are not required for item memory [11]. This assumption may explain why destination recall may be more prone to distortions than item memory or source memory.

\subsection{Destination memory and executive function}

Another cognitive domain that may be important for the fallibility of destination memory is executive function. Decline in episodic memory, and especially in source recall, is widely observed in patients with executive deficits. Schacter et al. [12] found that patients with executive dysfunction after prefrontal cortex damage were able to remember that information was previously encountered, but showed substantial difficulties in recalling where they had learned the information. These outcomes were replicated by studies showing a significant correlation between difficulties in source memory and executive dysfunction in healthy older people (e.g., [13-15]), individuals with Parkinson's disease [16], and people with Alzheimer's disease [17] (for a comprehensive review on the relationship between executive dysfunction and source memory deterioration, see, [18]). In line with this notion, destination memory function also correlated with executive function, especially with inhibition [1]. Inhibition, a core executive function, allows for the suppression of distracting information from ongoing processing, which contributes to selective attention ability [19]. Destination memory is likely to rely heavily on inhibitory processes in order to keep the focus of attention on the characteristics of the information (e.g., did tell you about the weekend?), destination (e.g., did I tell you about the weekend?), and/or selfmonitoring processes (e.g., did / tell you about the weekend?)

\subsection{Destination memory and self- referential processes}

According to Gopie et al. [3,5], when outputting information, one's main attentional focus is on oneself. This self-focus is argued to leave fewer attentional resources available to attribute information to its destination, which may account for the vulnerability of destination memory where typically the focus lies on other people. This assumption was tested by Gopie and MacLeod [3] who found that it is possible to reduce destination memory distortions by explicitly shifting participants' attention from themselves to the person to whom the information is outputted. In other words, decreasing self-focus by directing attention to the destination improved destination memory, an outcome suggesting self-focus as an important destination memory component. Another factor that may mediate the relationship between destination memory and the self is the autobiographical nature of destination memory. That is, destination memory is constructed in a specific spatiotemporal context with reference to oneself as a participant in the episode [3], a consideration that is in line with the autobiographical model of Conway [20] suggesting the self as a basic component of autobiographical recall. By highlighting the self-component of destination memory, the present review will attempt to advance some hypotheses about the neuroanatomical basis of destination memory.

\section{Neuroanatomical speculations}

Empirical research thus suggests that several cognitive factors may determine destination memory functioning; factors including episodic processing, executive function, and self-referential processes. To the best of our knowledge, there is no literature investigating the neural underpinning of destination recall. However, the aforementioned processes, illustrated in Figure 1, shape our hypothesis about the neuroanatomical basis of destination memory.

\subsection{Medial temporal lobes}

Since destination memory can be considered as an episodic component that enables associations between information and its episodic context [6], it is likely to involve brain areas that are responsible for associative mechanisms, such as the medial temporal lobe (MTL). The MTL has been widely described as a system of regions that significantly contribute to episodic memory, including the hippocampal formation, perirhinal cortex, parahippocampal cortex and entorhinal cortex [21]. In this extended "MTL memory system", neuroimaging studies have suggested a crucial role of hippocampus in relational memory [22]. Also, hippocampal involvement has been demonstrated in binding individual features into complex episodic memories during encoding and retrieving context information (e.g., [23-26], for a review on the hippocampal involvement in context memory, see [27]). The hippocampal involvement in context processing is of crucial interest to the present review, since destination memory contributes to context recall [6], which would require hippocampal involvement in destination memory. Further support for this assumption can be found in work by Mayes et al. [26] who proposed that the hippocampus is implied in across-domain associations (e.g., itemlocation). In a similar vein, studies suggest 
that the hippocampus is involved, possibly in conjunction with entorhinal cortex, whenever information is bound or associated with episodic context [28-30], as is the case for destination memory.

MTL implication in item and context association can also be illustrated by reference to the Binding of Items and Contexts model [31]. This model, built upon a framework proposed by Squire et al. [21], proposes different roles for the perirhinal and parahippocampal cortex, and the hippocampus proper. According to this model, the perirhinal cortex processes information about the qualities of objects ("what"), as outputted by neocortical areas, and the parahippocampal cortex process spatial information ("where"), as outputted by the posterior parietal cortex. The perirhinal and parahippocampal cortex project to the lateral and medial entorhinal areas respectively, and theseareas'inputs converge in the hippocampus (for a similar view, see $[32,33])$. In line with this model, research suggests that the perirhinal cortex processes information about items (e.g., what), whereas the parahippocampal cortex processes contextual information (e.g., where and when), and the hippocampus binds these items and contextual features [24,31,34]. Taken together, the MTL plays an important role in processing contextual information, and is likely to be involved in destination memory.

\subsection{Prefrontal cortex}

As mentioned above, early neuropsychological work suggested that lesions in the prefrontal cortex (PFC) may also disrupt episodic memory $[12,35]$. Also, a large body of fMRI literature has found that contextual judgments (i.e., source memory) are associated with activity in left lateral PFC, including the superior, middle, and inferior frontal gyrus (Brodmann areas 9, 10, 44, 46, and 47). This activity was observed for various types of contextual information (e.g., location) and modalities (e.g., auditory and visual stimuli) [36-38] (for a comprehensive review, see [39]). In a parallel manner, context recall has also been associated with right lateral PFC activation, especially in tasks requiring heuristic judgments, or decisions based on metacognitive expectations [40,41-43]. Another hypothesis about the functional specificity of the PFC in context memory has been proposed by Mitchell and Johnson [39]. According to this view, the ventrolateral PFC tends to be more involved in the encoding of specific item features, whereas the dorsolateral PFC tends to be more implied in control processes aimed at the organization and relations between contextual features (for a similar view, see, [43]). So far, these studies have focused on source memory. While it can be hypothesized that the same brain regions may be important in destination memory, this remains to be empirically studied.

\subsection{Precuneus}

Since destination memory is thought to involve self-referential processes, it may also depend on brain areas that are associated with these processes. Among the cortical midline structures, the precuneus is widely suggested to support self-referential processing, such as integration of self-relevant information with past experiences [44], construction of one's own visual perspective [45], judgments on one's own personality traits [46], judgments on self-descriptive [47], and mental simulation of self-generated actions [48]. In line with these findings, activation in the precuneus has been observed during autobiographical recall (e.g [49]), which also relies heavily on self-referential processes [20]. According to Cavanna and Trimble [50], the precuneus is an essential part of the neural network underpinning self-awareness and selfprocessing operations, which contributes to first-person perspective taking and experiences of agency. The latter suggestion is of crucial interest since destination memory is a memory system that primary deals with selfinitiated information.

\section{Destination memory: open questions}

In memory literature, research used to deal with context recall by referring to spatiotemporal aspects such as who, when, and where an event has took place. This literature can be enriched by investigating the "to whom" aspect, a contextual facet that is primarily concerned by the sense of agency and the interaction with our environment. Retaining the terminal of our thoughts and actions grants elaborating the appropriate response to our query would thus save valuable time and efforts. Another consequence of destination memory distortions is redundancy, or the tendency to repeat the same information to the same receiver. Such a distortion may induce several costs, such as increasing inferences and decreasing the amount of new information; bias that may attenuate communicative efficacy and, consequently, our social interactions with others.

As the present review tends to suggest, the vulnerability of destination memory can be attributed to its high demand on cognitive resources, namely, episodic processing, executive function, and self-referential processes (see Figure 2 for a summary). Although prominent, some questions remain open. First, the underlying neurocognitive mechanisms need further investigation by functional neuroimaging and lesion studies. Second, future research should focus on the dissociation between destination memory and more general episodic memory processes, such as item-context binding or autobiographical recall. While promising, research on destination memory is still in its infancy, and further studies are needed to reveal the exact cognitive and neuroanatomical basis of destination recall.

- Destination memory allows successful association between the information and its receiver, enhancing communicative efficacy and, consequently, our social interactions.

- This memory is found to be subject to distortions in younger adults, older adults, and patients with Alzheimer's disease.

- Destination recall is found to rely on episodic processes, executive function, and self-referential processes.

- This memory is likely to imply this memory may imply brain areas, such as medial temporal lobes (especially, hippocampus, perirhinal cortex, parahippocampal cortex, and entorhinal cortex), frontal lobes, and precuneus

Figure 2. Highlights of key points. 
[1] El Haj M., Postal V., Allain P., Destination memory in Alzheimer's disease: when I imagine telling Ronald Reagan about Paris, Cortex, 2013, 49, 82-89

[2] El Haj M., Postal V., Le Gall D., Allain P., Destination memory in mild Alzheimer's disease, Behav. Neurol., 2013, 26, 215-216

[3] Gopie N., MacLaeod C.M., Destination memory: stop me if I've told you this before, Psychol. Sci., 2009, 20, 1492-1499

[4] El Haj M., Fasotti L., Allain P., Destination memory for emotional information, Exp. Aging Res., accepted for publication

[5] Gopie N., Craik F.l., Hasher L., Destination memory impairment in older people, Psychol. Aging, 2010, 25, 922-928

[6] El Haj M., Moroni C., Luyat M., Omigie D., Allain P., To what extent does destination recall induce episodic reliving? Evidence from Alzheimer's disease, J. Clin. Exp. Neuropsychol., 2014, 36, 127-136

[7] Dudukovic N.M., Knowlton B.J., Remember-Know judgments and retrieval of contextual details, Acta Psychol. (Amst.), 2006, 122, 160173

[8] Gardiner J.M., Ramponi C., Richardson-Klavehn A., Experiences of remembering, knowing, and guessing, Conscious. Cogn., 1998, 7, $1-26$

[9] Wheeler M.A., Stuss D.T., Tulving E., Toward a theory of episodic memory: the frontal lobes and autonoetic consciousness, Psychol. Bull., 1997, 121, 331-354

[10] Grober E., Buschke, H. Genuine memory deficits in dementia, Dev. Neuropsychol., 1987, 3, 13-36

[11] El Haj M., Kessels R.P., Context memory in Alzheimer's disease, Dement. Geriatr. Cogn. Dis. Extra, 2013, 3, 342-350

[12] Schacter D.L., Harbluk J.L., McLachlan D.R., Retrieval without recollection: an experimental analysis of source amnesia, J. Verbal Learn. Verbal Behav., 1984, 23, 593-611

[13] El Haj M., Allain P., Relationship between source monitoring in episodic memory and executive function in normal aging, Geriatr. Psychol. Neuropsychiatr. Vieil., 2012, 10, 197-205

[14] Glisky E.L., Rubin S.R., Davidson P.S., Source memory in older adults: an encoding or retrieval problem?, J. Exp. Psychol. Learn. Mem. Cogn., 2001, 27, 1131-1146

[15] Glisky E.L., Kong L.L., Do young and older adults rely on different processes in source memory tasks? A neuropsychological study, J. Exp. Psychol. Learn. Mem. Cogn., 2008, 34, 809-822

[16] Drag L.L., Bieliauskas L.A., Kaszniak A.W., Bohnen N.I., Glisky E.L., Source memory and frontal functioning in Parkinson's disease, J. Int. Neuropsychol. Soc., 2009, 15, 399-406

[17] El Haj M., Fasotti L., Allain P., Source monitoring in Alzheimer's disease, Brain Cogn., 2012, 80, 185-191

[18] El Haj M., Allain P., What do we know about the relationship between source monitoring deficits and executive dysfunction?, Neuropsychol. Rehabil., 2012, 22, 449-472

[19] Miyake A., Friedman N.P., Emerson M.J., Witzki A.H., Howerter A., Wager T.D., The unity and diversity of executive functions and their contributions to complex "frontal lobe" tasks: a latent variable analysis, Cogn. Psychol., 2000, 41, 49-100

[20] Conway M.A., Memory and the self, J. Mem. Lang., 2005, 53, 594-628

[21] Squire L.R., Knowlton B., Musen G., The structure and organization of memory, Annu. Rev. Psychol., 1993, 44, 453-495

[22] Lepage M., Habib R., Tulving E., Hippocampal PET activations of memory encoding and retrieval: the HIPER model, Hippocampus, 1998, 8, 313-322

[23] Davachi L., Item, context and relational episodic encoding in humans, Curr. Opin. Neurobiol., 2006, 16, 693-700

[24] Diana R.A., Yonelinas A.P., Ranganath C., Imaging recollection and familiarity in the medial temporal lobe: a three-component model, Trends Cogn. Sci., 2007, 11, 379-386

[25] Henson R., A mini-review of fMRI studies of human medial temporal lobe activity associated with recognition memory, Q. J. Exp. Psychol. B, 2005, 58, 340-360

[26] Mayes A., Montaldi D., Migo E., Associative memory and the medial temporal lobes, Trends Cogn. Sci., 2007, 11, 126-135

[27] Kessels R.P., Kopelman M.D., Context memory in Korsakoff's syndrome, Neuropsychol. Rev., 2012, 22, 117-131

[28] Bird C.M., Burgess N., The hippocampus and memory: insights from spatial processing, Nat. Rev. Neurosci., 2008, 9, 182-194

[29] Lipton P.A., Eichenbaum H., Complementary roles of hippocampus and medial entorhinal cortex in episodic memory, Neural Plast., 2008,258467

[30] Shohamy D., Wagner A.D., Integrating memories in the human brain: hippocampal-midbrain encoding of overlapping events, Neuron, 2008, 60, 378-389

[31] Eichenbaum H., Yonelinas A.P., Ranganath C., The medial temporal lobe and recognition memory, Annu. Rev. Neurosci., 2007, 30, 123152

[32] Kerr K.M., Agster K.L., Furtak S.C., Burwell R.D., Functional neuroanatomy of the parahippocampal region: the lateral and medial entorhinal areas, Hippocampus, 2007, 17, 697-708

[33] Witter M.P., Naber P.A., van Haeften T., Machielsen W.C., Rombouts S.A., Barkhof F., et al., Cortico-hippocampal communication by way of parallel parahippocampal-subicular pathways, Hippocampus, 2000, 10, 398-410

[34] Ranganath C., Binding items and contexts the cognitive neuroscience of episodic memory, Curr. Dir. Psychol. Sci., 2010, 19, 131-137

[35] Shimamura A.P., Squire L.R., A neuropsychological study of fact memory and source amnesia, J. Exp. Psychol. Learn. Mem. Cogn., $1987,13,464-473$

[36] Dobbins I.G., Han S., Cue- versus probe-dependent prefrontal cortex activity during contextual remembering, J. Cogn. Neurosci., 2006, 18, 1439-1452

[37] Dudukovic N.M., Wagner A.D., Goal-dependent modulation of declarative memory: neural correlates of temporal recency decisions and novelty detection, Neuropsychologia, 2007, 45, 2608-2620 
[38] Simons J.S., Gilbert S.J., Owen A.M., Fletcher P.C., Burgess P.W., Distinct roles for lateral and medial anterior prefrontal cortex in contextual recollection, J. Neurophysiol., 2005, 94, 813-820

[39] Mitchell K.J., Johnson M.K., Source monitoring 15 years later: what have we learned from $\mathrm{fMRI}$ about the neural mechanisms of source memory?, Psychol. Bull., 2009, 135, 638-677

[40] Kensinger E.A., Clarke R.J., Corkin S., What neural correlates underlie successful encoding and retrieval? A functional magnetic resonance imaging study using a divided attention paradigm, J. Neurosci., 2003, 23, 2407-2415

[41] Mitchell K.J., Johnson M.K., Raye C.L., Greene E.J., Prefrontal cortex activity associated with source monitoring in a working memory task, J. Cogn. Neurosci., 2004, 16, 921-934

[42] Raye C.L., Johnson M.K., Mitchell K.J., Nolde S.F., D'Esposito M., fMRI investigations of left and right PFC contributions to episodic remembering, Psychobiology, 2000, 28, 197-206

[43] Staresina B.P., Davachi L., Differential encoding mechanisms for subsequent associative recognition and free recall, J. Neurosci., 2006, 26, 9162-9172

[44] Summerfield J.J., Hassabis D., Maguire E.A., Cortical midline involvement in autobiographical memory, Neuroimage, 2009, 44, 1188-1200
[45] Freton M., Lemogne C., Bergouignan L., Delaveau P., Lehéricy S., Fossati P., The eye of the self: precuneus volume and visual perspective during autobiographical memory retrieval, Brain Struct. Funct., 2013, 1-10

[46] Kircher T.T., Senior C., Phillips M.L., Benson P.J., Bullmore E.T., Brammer M., et al., Towards a functional neuroanatomy of self processing: effects of faces and words, Cogn. Brain Res., 2000, 10, 133-144

[47] Kircher T.T., Brammer M., Bullmore E., Simmons A., Bartels M., David A.S., The neural correlates of intentional and incidental self processing, Neuropsychologia, 2002, 40, 683-692

[48] Ruby P., Decety J., Effect of subjective perspective taking during simulation of action: a PET investigation of agency, Nat. Neurosci., 2001, 4, 546-550

[49] Addis D.R., McIntosh A.R., Moscovitch M., Crawley A.P., McAndrews M.P., Characterizing spatial and temporal features of autobiographical memory retrieval networks: a partial least squares approach, Neuroimage, 2004, 23, 1460-1471

[50] Cavanna A.E., Trimble M.R., The precuneus: a review of its functional anatomy and behavioural correlates, Brain, 2006, 129, 564-583 